DETERMINANTES DE ESTRUTURA DE CAPITAL DE EMPRESAS DE CAPITAL ABERTO DO SETOR ELÉTRICO NO BRASIL: UMA ABORDAGEM BASEADA NA TRADE-OFF THEORY E NA PECKING ORDER THEORY

Versão do autor aceita publicada online: 06 dez. 2021

Publicado online: 06 jan. 2022

Como citar esse artigo - American Psychological Association (APA): Fodra, M. (2022). Determinantes de estrutura de capital de empresas de capital aberto do setor elétrico no Brasil: uma abordagem baseada na Trade-Off Theory e na Pecking Order Theory. Exacta. DOI: https://doi.org/10.5585/exactaep.2022.20823.

\title{
Marcelo Fodra
}

https://orcid.org/0000-0003-0905-6580

Universidade Federal de Uberlândia

Professor do Departamento de Finanças da Faculdade de Gestão e Negócios da Universidade Federal de Uberlândia.

Contato principal para correspondência.

\section{RESUMO:}

O objetivo deste trabalho foi avaliar os determinantes de estrutura de capital das empresas de capital aberto do setor elétrico brasileiro, listadas na Bolsa de Valores de São Paulo, em relação ao endividamento de curto prazo, longo prazo e geral. Foi coletada uma amostra composta por dados contábeis anuais de 80 empresas, privadas e estatais, atuantes no setor elétrico brasileiro entre 2009 e 2019 , empregando-se o método de regressões lineares múltiplas, com dados em painel, usando efeitos fixos e efeitos aleatórios. Os resultados apontaram que os determinantes de estrutura de capital lucratividade, oportunidade de crescimento e liquidez tiveram comportamento melhor alinhados à POT, ao passo que o risco se apresentou melhor explicado pela TOT e pela POT.

PALAVRAS CHAVE: Fontes de financiamento; Endividamento; Modelagem com dados em painel; Estrutura de capital. Privatização.

\section{DETERMINANTS OF CAPITAL STRUCTURE OF OPEN CAPITAL COMPANIES IN THE ELECTRIC SECTOR IN BRAZIL: AN APPROACH BASED ON TRADE-OFF THEORY AND ON PECKING ORDER THEORY}

\section{ABSTRACT:}

The objective of this work was to evaluate the determinants of capital structure of publicly traded companies in the Brazilian electricity sector, listed on the São Paulo Stock Exchange, related to short-term, long-term and general indebtedness. A sample consisting of annual accounting data from 80 private and state-owned companies operating in the Brazilian electricity sector between 2009 and 2019 was collected, using the method of multiple linear regressions, with panel data using fixed effects and random effects. The results showed that the 
determinants of capital structure, profitability, growth opportunity and liquidity had a behavior better aligned with the POT, while the risk was better explained by the TOT and POT.

KEY WORDS: Financing source; Indebtedness; Modeling with panel data; Capital structure. Privatization.

\section{Introdução}

As decisões sobre a seleção de fontes de financiamento das empresas é um assunto relevante e, nas últimas seis décadas, tem sido amplamente discutido, levando ao desenvolvimento de teorias sobre a estrutura de capital das organizações (Cevheroglu-Acar, 2018). Contudo, até o momento, inexistem abordagens simples capazes de explicar o comportamento dos agentes econômicos em suas decisões de escolha entre dívidas e capital próprio (Vo, 2017).

O início das discussões sobre o financiamento da empresa se deu com o trabalho de Modigliani and Miller (1958), no qual os autores afirmavam que, sob certas condições específicas, a proporção entre dívidas e capital próprio era irrelevante para o valor da firma (Bastos, Nakamura \& Basso, 2009). Posteriormente, os mesmos Modigliani and Miller (1963) reconheceram que o endividamento pode afetar o valor das empresas, em razão de benefícios fiscais gerados pelo uso do capital de terceiros (Brito, Corrar \& Batistella, 2007), incentivando diversos estudos teóricos para explicar as decisões de financiamento das empresas, destacando-se: Miller (1977) e DeAngelo and Masulis (1980), Myers (1984), Myers and Majluf (1984), dentre outros (Brito et al, 2007).

Com o advento da Revolução Industrial, o uso intensivo de máquinas na produção e a urbanização induziram a melhoria da qualidade de vida das pessoas, fazendo aumentar a demanda mundial por energia (Aydin, 2019), impondo que o funcionamento das empresas e o crescimento econômico passassem a ter, em boa parte, a energia como insumo fundamental. Segundo Fotourehchi (2017, as cited in Tsaurai and Ngcobo 2020), a energia é fator importante no desenvolvimento econômico de um país, impactando as tarefas do dia-a-dia e também as atividades industriais, existindo uma correlação positiva entre a oferta de energia elétrica e o desenvolvimento econômico de uma sociedade (Goldemberg \& Lucon, 2008). O setor elétrico supre os demais segmentos da atividade econômica com um insumo básico à produção (Montoya et al., 2013). Também há elementos que apontam a ocorrência de correlação causal bidirecional entre o consumo de energia e o aumento do PIB (Belke, Dreger \& Haan 2010). Segundo Ouedraogo (2013) e Niu et al., (2013) há igualmente evidências de 
correlação positiva de longo prazo entre o consumo de energia elétrica e o Índice de Desenvolvimento Humano - IDH.

Segundo Montoya, Pasqual, Lopes e Guilhoto (2013), a demanda por energia elétrica no Brasil deveria aumentar em virtude do incremento da atividade empresarial e do aumento populacional esperados em períodos futuros. Portanto, o consumo de energia define o perfil de desenvolvimento de um país, gerando associações entre o consumo de energia elétrica, o crescimento econômico e a qualidade de vida das pessoas (Niu, et al., 2013).

O setor elétrico brasileiro passou por intenso processo de privatização durante os anos 1990 com marcante presença de capital estrangeiro (Serva, 2003), o que produziu um setor elétrico híbrido, com a presença de empresas de capital privado e estatal, atuantes nas atividades de geração, distribuição, transmissão e comercialização de energia elétrica (Ganim, 2008).

O cenário exposto ressalta a importância da energia para o funcionamento das empresas e a manutenção dos níveis de qualidade de vida das populações, reforçando a relevância estratégica de existir um setor elétrico eficiente nos sistemas econômicos, pois ele é o encarregado de produzir um insumo essencial para a atividade econômica e o bem-estar social. Portanto, estudos que abordem aspectos estratégicos do setor elétrico são importantes para a compreensão da lógica de funcionamento deste setor econômico.

É sabido que, desde a proposição da irrelevância da estrutura de capital, feita por Modigliani e Miller (1958), diversos trabalhos acadêmicos foram produzidos, na tentativa de construir teorias capazes de explicar o comportamento dos agentes no que diz respeito às decisões de financiamento. Além disso, pesquisas empíricas discutiram o papel dos determinantes da estrutura de capital das firmas, encontrando resultados variados.

Pesquisas de natureza empírica, versando sobre os determinantes de estrutura de capital foram elaborados em diversos países, como por exemplo: Vo (2017), Cevheroglu-Acar (2018), Jarallah, Saleh and Salim (2018), Kadek and Bagus (2019), Bhama, Jain and Yadav (2019), Martinez, Scherger and Guercio (2019). Além dos estudos mencionados, existiram trabalhos que investigaram questões sobre estrutura de capital de empresas brasileiras, tais como: Perobelli e Famá (2003), Brito et al., (2007), Bastos et al., (2009), Albanez e Valle (2009), Lima e Brito (2010) e Rodrigues, Moura, Santos and Sobreiro (2017).

Embora exista expressiva quantidade de trabalhos versando sobre a estrutura de capital das empresas, sob diversos aspectos, poucos deles abordaram tais questões em relação ao setor 
elétrico brasileiro. Dentre os trabalhos nacionais que trataram do assunto, destacam-se: Doege e Matos (2011) e Kuroda, Moralles and Albuquerque (2019). Dessa maneira, a exposição anterior apresenta a lacuna de pesquisa que este trabalho se propõe a preencher: estudar os determinantes de estrutura de capital das empresas de capital aberto do setor elétrico brasileiro.

Portanto, o objetivo deste trabalho foi estudar os determinantes da estrutura de capital das empresas de capital aberto do setor elétrico brasileiro, com suas ações negociadas na Bolsa de Valores de São Paulo, relativos ao endividamento de curto prazo, longo prazo e geral, a partir das premissas da TOT e da POT, no período compreendido entre 2009 e 2019.

Dado que o setor elétrico brasileiro é um segmento de importância estratégica e apresenta peculiaridades, devido à convivência de empresas privadas e estatais em um único ambiente, este trabalho pretende oferecer contribuições teóricas para auxiliar na melhor compreensão dos mecanismos tratados na decisão de financiamento das empresas do segmento. Somado a isso, pretende-se contribuir com as análises empíricas, ao reconhecer os determinantes da estrutura de capital que influenciam na seleção das fontes de financiamento de um setor de atividade em específico, indicando seu eventual alinhamento à TOT ou POT.

O presente artigo foi estruturado em cinco tópicos, sendo este primeiro de natureza introdutória, no qual se faz a contextualização, apresenta-se o objetivo do trabalho e são expostas suas justificativas. O segundo tópico traz o referencial teórico que embasou as discussões, ao passo que, no terceiro item, apresentam-se as questões metodológicas usadas na elaboração do material. O quarto item apresenta e discute os resultados encontrados e o quinto, expõe as considerações finais da pesquisa.

\section{Referencial teórico}

\subsection{Abordagens teóricas sobre a estrutura de capital}

A discussão inicial sobre as decisões de financiamento das empresas e o seu valor teve início no trabalho de Modigliani and Miller (1958), com suas hipóteses restritivas. Embora, a partir dele, tenham surgido teorias para explicar as decisões de financiamento das organizações, o tema ainda parece longe de um consenso.

A pesquisa inicial de Modigliani and Miller (1958) propôs que, sob hipóteses específicas de um mercado perfeito, a política de dividendos das empresas, assim como as suas decisões de financiamento, são irrelevantes (Brealey, Myers \& Allen, 2013). Posteriormente, Modigliani 
and Miller (1963) reconheceram impactos sobre o valor da empresa decorrentes do aumento do endividamento e da possível anulação dos benefícios fiscais atrelados às dívidas. À medida que novas pesquisas surgiam, outros fatores foram incorporados, produzindo abordagens distintas para o tema: Miller (1977) tratou dos custos de falência, DeAngelo and Masulis (1980) estudaram os efeitos da tributação sobre as pessoas físicas e o impacto no capital próprio das empresas.

Segundo a Teoria de Agência, a empresa é formada por um conjunto de contratos, determinantes dos relacionamentos entre as partes envolvidas com a firma, enquanto a gestão dos conflitos ocasionados nestes relacionamentos geraria custos de agência (Jensen \& Meckling, 1976). Para Jensen (1986), a presença de fluxo de caixa livre poderia incentivar os gestores a tomarem decisões não ótimas, cabendo ao endividamento a função de disciplinar as ações dos gestores, em função do risco de falência. A partir da Teoria de Agência surgiu a Teoria do Trade-Off, ou Trade-Off Theory (TOT), propondo que a empresa busca uma combinação ótima entre as dívidas e o capital próprio, capaz de minimizar os custos e de equilibrar valor da firma (Albanez \& Valle, 2009).

Por outro lado, Ross (1977) afirmou que os gestores conhecem a verdadeira possibilidade de distribuição de lucros de uma empresa e, por isso, as escolhas feitas pelos administradores em relação à estrutura de capital sinalizam ao mercado informações internas da empresa. Firmas com maiores proporções de ações em poder de insiders sinalizariam ao mercado a existência de projetos com boa qualidade, refletindo na escolha de suas fontes de financiamento (Leland \& Pyle, 1977). Portanto, administradores podem deter mais informações internas do que os mercados e investidores, incentivando a elevação do endividamento da firma, produzindo assimetrias informacionais e estabelecendo uma preferência hierárquica em relação às fontes de financiamento das empresas (Myers \& Majluf, 1984).

Os argumentos propostos na Teoria da Assimetria Informacional embasam a Pecking Order Theory (POT), apontando que a hierarquia das fontes de financiamento começa pelos fundos internos (lucros retidos), seguidos pelas dívidas e, se necessário, recorrendo-se ao uso de capital próprio, por meio de emissões de novas ações (Myers, 1984). As empresas mais lucrativas deveriam ser menos endividadas, pois disporiam de fontes internas para financiamento de novos projetos, ao passo que as organizações menos lucrativas recorreriam a financiamentos externos para seus empreendimentos (Myers, 2001). 
De acordo com as premissas da POT, sob assimetria informacional, o custo de financiamento com dívidas superaria qualquer outro, impedindo a existência de uma estrutura ótima de capital, o que limitaria a capacidade de endividamento da empresa ao seu fluxo de caixa, fazendo-a preferir recursos internos aos demais (Lima \& Brito, 2010).

A POT também supõe que, além de não haver uma estrutura ótima de capital, a tensão entre os benefícios fiscais e os custos de falência são menos importantes, pois, a relação entre dívidas e capital próprio muda sempre que se desequilibram os fluxos de caixa internos da empresa, em razão das oportunidades reais de investimento ou dos dividendos pagos (ShyamSunder \& Myers, 1999).

\subsection{Evidências empíricas sobre a estrutura de capital das empresas}

A partir dos argumentos teóricos propostos, diversos trabalhos investigaram empiricamente os determinantes da estrutura de capital das empresas, levando em conta a aderência das firmas a uma dada teoria. No âmbito internacional, destacam-se os trabalhos seminais de Bradley, Jarrel and Kim (1984), Titman (1984), Titman and Wessels (1988), Harris and Raviv (1991), Rajan and Zingales (1995), dentre outros.

Além de pesquisas conduzidas em economias desenvolvidas, também existem trabalhos relevantes realizados em países em desenvolvimento, como é o caso de Booth, Aivazian, Demirguc-Kunt and Maksimovic (2001). Com escopo mais restrito, Shah and Hizaji (2004) fizeram estudos para empresas do Paquistão, enquanto Espinosa, Maquieira, Vieito and González (2012) trataram dos determinantes de estrutura de capital em países em desenvolvimento em geral, enquanto Huang and Song (2006) investigaram a estrutura de capital de firmas chinesas. Por sua vez, Vo (2017) analisou determinantes de estrutura de capital para empresas do Vietnã, ao passo que Cevheroglu-Acar (2018) fez o mesmo estudo para empresas da Turquia. Em adição, Jarallah, Saleh and Salim (2018) avaliaram os determinantes dá estrutura de capital de empresas no Japão, ao passo que Kadek and Bagus (2019) fizeram esta análise para firmas da Indonésia e Bhama, Jain and Yadav (2019) testaram as evidências da POT para empresas chinesas e indianas. Além disso, Martinez, Scherger and Guercio (2019) fizeram uma revisão sistemática sobre o uso de POT e TOT em relação à estrutura de capital de pequenas e médias empresas em geral.

No que tange às Américas e ao Brasil, destacam-se os trabalhos de: Perobelli e Famá (2002), Perobelli e Famá (2003), Brito et al., (2007), Bastos et al., (2009), Albanez e Valle (2009), Lima e Brito (2010), Correa, Basso e Nakamura (2013), além de Rodrigues, Moura, Santos 
and Sobreiro (2017). Contudo, apesar da abrangência do tema, são relativamente escassos os trabalhos que estudaram a estrutura de capital de empresas atuantes em setores regulados, especificamente o setor elétrico. Neste quesito, Spiegel and Spulber (1994) analisaram questões sobre a estrutura de capital de empresas regulamentadas por órgãos e agências de controle em contexto geral, enquanto Md-Yusuf, Yunus and Supaat (2013) enfocaram seus estudos no setor elétrico da Malásia. Além deles, Mudany, Letting and Gituro (2020) abordaram questões estratégicas sobre a estrutura de capital de empresas no Quênia, enquanto Bagais, Aljaaidi and Alothman (2021) focalizaram o endividamento de curto e longo prazo e o valor de empresas do setor elétrico da Arábia Saudita.

No cenário brasileiro, Ferreira, Bertucci e Pereira (2010) averiguaram a estrutura de capital e os investimentos de empresas brasileiras do setor de energia e telecomunicações, enquanto Sant 'Ana e Silva (2015) esquadrinharam os determinantes da estrutura de capital de empresas brasileiras do setor de tecnologia. Além dos mencionados, Doege e Matos (2011) sondaram o eventual padrão de comportamento na estrutura de capital de distribuidoras de energia no Brasil e, mais recentemente, Kuroda, Moralles and Albuquerque (2019) analisaram a alavancagem financeira e a maturação de investimentos realizados em firmas brasileiras do setor elétrico.

\section{METODOLOGIA}

\subsection{Caracterização da pesquisa e fonte de dados}

Este trabalho pode ser caracterizado como uma pesquisa quantitativa, do tipo descritiva, que se utilizou de dados secundários. Uma pesquisa descritiva é aquela na qual ocorre a observação, registro, análise e correlação de elementos, com a finalidade de identificar formas, estruturas e conteúdos, a partir dos elementos estudados (Cervo \& Bervian, 2002).

Foram utilizados dados contábeis anuais das empresas do setor elétrico brasileiro, divulgados por firmas de capital aberto, que possuíam ações negociadas na Bolsa de Valores de São Paulo. Os dados foram coletados na base Economatica e abrangeram o período entre 2009 e 2019, considerados em valores históricos.

A amostra foi composta pelas 80 empresas de capital aberto ativas, listadas na Bolsa de Valores, operantes no setor elétrico brasileiro no momento da pesquisa. Optou-se por utilizar a totalidade das empresas que se encontravam ativas no momento da coleta dos dados, descartando-se aquelas que se achavam inativas na oportunidade. A seleção das empresas 
componentes da amostra foi feita mediante uso de amostragem por conveniência. Triola (2012, p. 655) afirma que a amostragem por conveniência é aquela "na qual os elementos são selecionados por estarem facilmente disponíveis". Para que fizesse parte da amostra, era necessário que a empresa tivesse produzido informações usadas para o cálculo de todos os índices usados neste trabalho em ao menos um dos exercícios no período pesquisado. Foram excluídas da amostra as observações que apresentaram Patrimônio Líquido negativo.

\subsection{Definição das variáveis utilizadas}

As variáveis dependentes examinadas neste trabalho foram: Endividamento de Curto Prazo (ECP), Endividamento de Longo Prazo (ELP) e Endividamento Geral (EGE). As variáveis independentes, representando proxies dos determinantes de estrutura de capital das empresas foram: lucratividade, risco de falência, tamanho, tangibilidade, oportunidades de crescimento, liquidez e benefícios fiscais não decorrentes de endividamento. O Quadro 1 mostra os embasamentos teóricos para uso de cada variável, bem como suas formas de apuração e embasamento referencial.

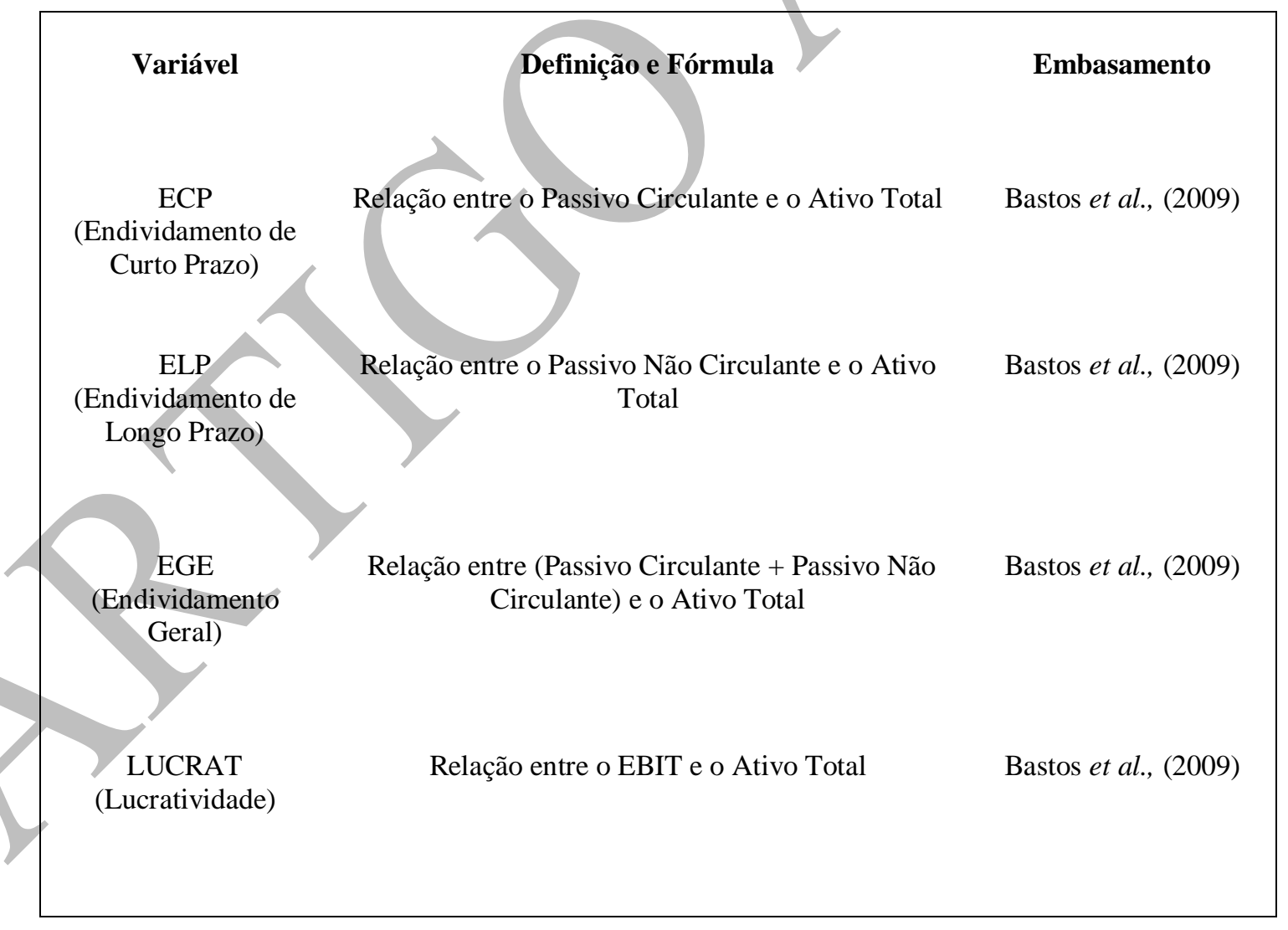




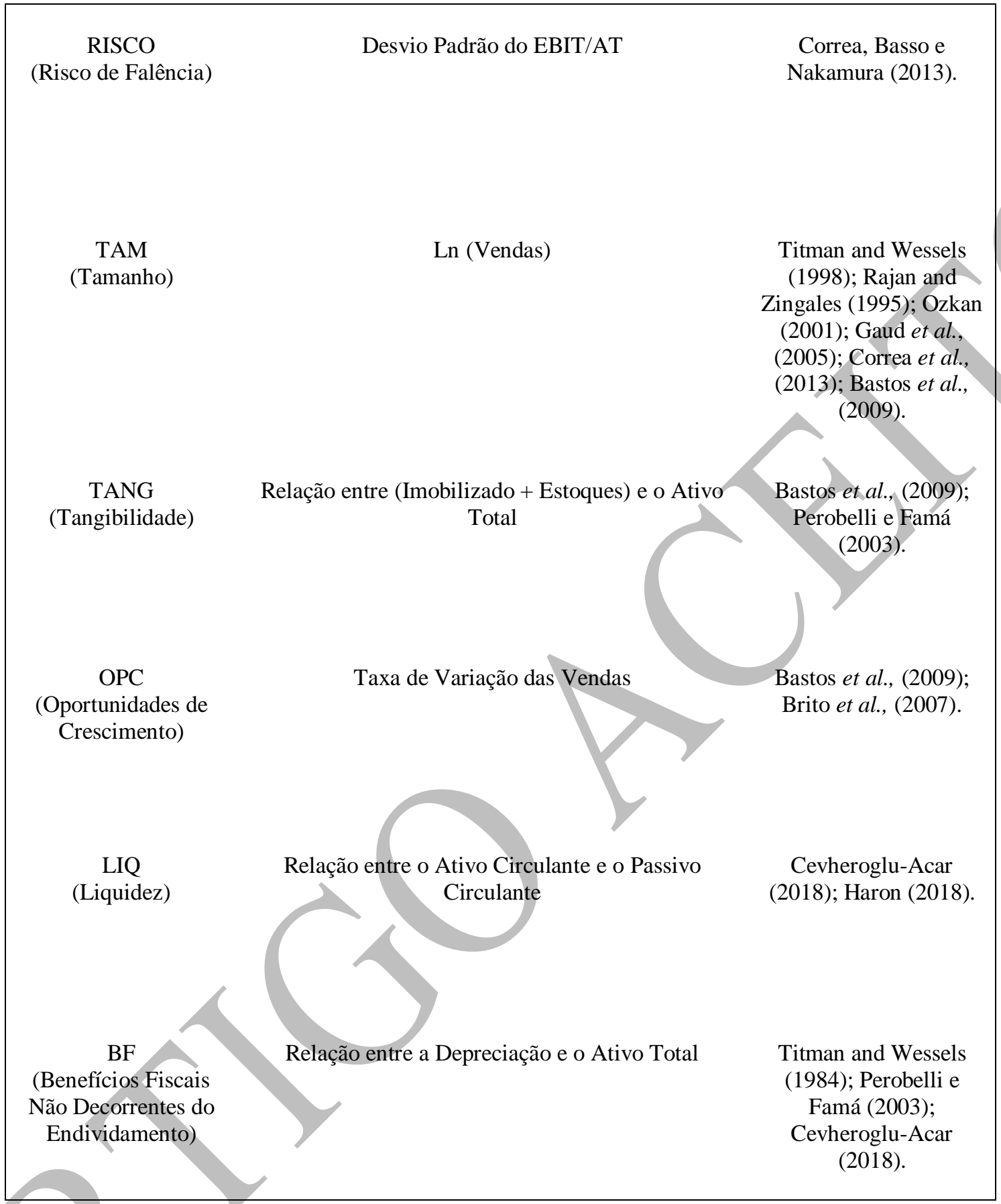

Figura 1: Descrição das variáveis utilizadas na pesquisa. (Fonte: Autor).

\subsection{Procedimentos econométricos}

Para captar a dimensão longitudinal dos dados coletados, optou-se pela utilização de regressões múltiplas com dados em painel. Um conjunto longitudinal, ou em painel, considera uma amostra de indivíduos em diversos períodos de tempo, levando em conta os efeitos econômicos que não poderiam ser identificados apenas em cross-sections ou em séries temporais (Pindyck \& Rubinfeld, 2004), permitindo o controle adequado da heterogeneidade individual (Baltagi, 1998). 
As variáveis coletadas viabilizaram a construção de uma base de dados em um painel desbalanceado. Nos painéis balanceados, todos os indivíduos possuem as mesmas quantidades de observações ao longo do tempo, enquanto em painéis desbalanceados, algum indivíduo pode ter quantidades diferentes de observações (Greene, 2008).

A presença de variações em painel foi apurada mediante a aplicação do Teste LM BreuschPagan, o qual verifica se os componentes individuais de variância dos indivíduos ou de unidades de tempo são nulos, apontando que o modelo de regressão com efeitos aleatórios lida melhor com a heterogeneidade da amostra do que outra modelagem baseada em Mínimos Quadrados Ordinários (MQO) (Tiryaki, 2017).

Feita a verificação da presença de efeitos de painel, foi aplicado o Teste Robusto de Hausman, proposto por Cameron and Trivedi (2010), com o propósito de avaliar se o melhor ajuste se dá por meio de efeitos fixos ou aleatórios. A aplicação de regressões com dados em painel com efeitos fixos determina que cada indivíduo presente na amostra possua um intercepto próprio (Kennedy, 2009). A hipótese nula do teste afirma que a modelagem mais adequada é com efeitos aleatórios, enquanto a sua rejeição, define que o melhor ajuste se dá com efeitos fixos (Wooldridge, 2016). O Teste de Schaffer e Stillman foi aplicado para confirmar os resultados obtidos no Teste Robusto de Hausman. Utilizou-se a estratificação ao nível de indivíduos, conforme proposto por Bastos e Nakamura (2009), para tornar as regressões robustas em relação à heteroscedasticidade.

As análises do endividamento propostas foram elaboradas de acordo com as equações a seguir:

$\mathrm{ECP}_{\mathrm{i}, \mathrm{t}}=\beta_{0}+\beta_{1} \mathrm{LUCRAT}_{\mathrm{i}, \mathrm{t}}+\beta_{2} \mathrm{RISCO}_{\mathrm{i}, \mathrm{t}}+\beta_{3} \mathrm{TAM}_{\mathrm{i}, \mathrm{t}}+\beta_{4} \mathrm{TANG}_{\mathrm{i}, \mathrm{t}}+\beta_{5} \mathrm{OPC}_{\mathrm{i}, \mathrm{t}}+\beta_{6} \mathrm{LIQ}_{\mathrm{i}, \mathrm{t}}+\beta_{7} \mathrm{BF}_{\mathrm{i}, \mathrm{t}}+\mu_{\mathrm{i}, \mathrm{t}} \quad$ (Equação

$\operatorname{ELP}_{\mathrm{i}, \mathrm{t}}=\beta_{0}+\beta_{1} \mathrm{LUCRAT}_{\mathrm{i}, \mathrm{t}}+\beta_{2} \mathrm{RISCO}_{\mathrm{i}, \mathrm{t}}+\beta_{3} \mathrm{TAM}_{\mathrm{i}, \mathrm{t}}+\beta_{4} \mathrm{TANG}_{\mathrm{i}, \mathrm{t}}+\beta_{5} \mathrm{OPC}_{\mathrm{i}, \mathrm{t}}+\beta_{6} \mathrm{LIQ}_{\mathrm{i}, \mathrm{t}}+\beta_{7} \mathrm{BF}_{\mathrm{i}, \mathrm{t}}+\mu_{\mathrm{i}, \mathrm{t}} \quad$ (Equação 2)

$\mathrm{EGE}_{\mathrm{i}, \mathrm{t}}=\beta_{0}+\beta_{1} \mathrm{LUCRAT}_{\mathrm{i}, \mathrm{t}}+\beta_{2} \mathrm{RISCO}_{\mathrm{i}, \mathrm{t}}+\beta_{3} \mathrm{TAM}_{\mathrm{i}, \mathrm{t}}+\beta_{4} \mathrm{TANG}_{\mathrm{i}, \mathrm{t}}+\beta_{5} \mathrm{OPC}_{\mathrm{i}, \mathrm{t}}+\beta_{6} \mathrm{LIQ}_{\mathrm{i}, \mathrm{t}}+\beta_{7} \mathrm{BF}_{\mathrm{i}, \mathrm{t}}+\mu_{\mathrm{i}, \mathrm{t}}$ (Equação

\section{RESULTADOS E DISCUSSÃO}

Inicialmente, pode-se observar que, exceto em relação ao tamanho da firma, as variáveis independentes mostraram elevados níveis de dispersão em relação às suas médias, reflexo da diversidade entre os indivíduos presentes na amostra. No setor elétrico brasileiro convivem 
empresas dedicadas à geração, transmissão, distribuição e comercialização de energia, privadas e estatais, cada qual com suas especificidades.

Foram apurados valores mínimos negativos em lucratividade, risco e oportunidades de crescimento, em virtude das características próprias destas variáveis, o que não ocorreu em relação ao tamanho, tangibilidade, liquidez e benefício fiscal não derivado das dívidas, tendo em vista que tais variáveis somente podem assumir valores nulos ou positivos. Os elementos expostos podem ser vistos na Tabela 1:

Tabela 1 - Estatísticas descritivas e matriz de correlações das variáveis independentes:

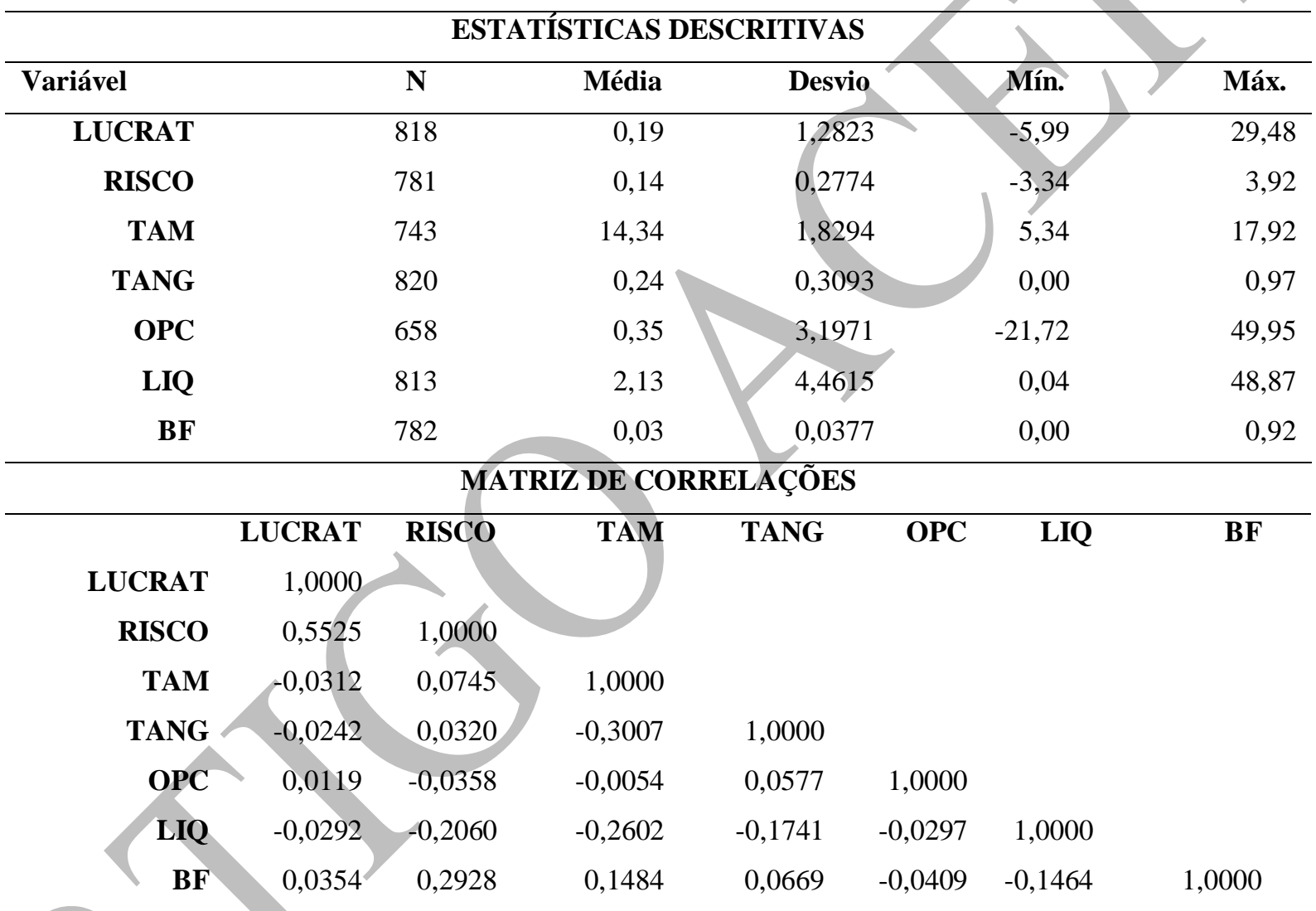

Fonte: Elaborado pelo autor.

Conforme se verifica na mesma Tabela 1, o único par de variáveis que apresentou correlação linear superior a 0,5 foi lucratividade e risco, o qual obteve correlação linear positiva semiforte, mas não causou multicolinearidade capaz de afetar a qualidade da modelagem econométrica. O coeficiente VIF (Fator de Inflação de Variância) médio calculado foi de 1,35, reforçando a evidência de ausência de multicolinearidade entre as variáveis utilizadas nos modelos. O VIF indica o efeito que outras variáveis independentes exercem sobre o erropadrão de um coeficiente de regressão, não devendo assumir valores maiores do que 10,00 (Hair, Black, Babin, Anderson \& Tatham, 2009). 
A seguir, foi aplicado o Teste LM Breusch-Pagan, para detecção de eventuais efeitos de painel na amostra, conforme os resultados expostos na Tabela 2:

Tabela 2 - Resultados da aplicação do Teste LM Breusch-Pagan e dos testes de Hausman e Schaffer e Stillman aos modelos de endividamento

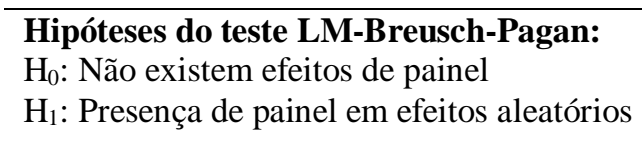

\begin{tabular}{|c|c|c|}
\hline $\begin{array}{l}\chi^{2}=329,64 \\
\text { Valor-P }=0,0000 \\
\text { Rejeita-se } \mathrm{H}_{0}\end{array}$ & $\begin{array}{c}\chi^{2}=776,52 \\
\text { Valor-P }=0,0000 \\
\text { Rejeita-se } \mathrm{H}_{0}\end{array}$ & $\begin{array}{r}\chi^{2}= \\
\text { Valor- } \\
\text { Reje }\end{array}$ \\
\hline \multicolumn{3}{|c|}{$\begin{array}{l}\text { Hipóteses do Teste Robusto de Hausman e do Teste de Sc } \\
\mathrm{H}_{0} \text { : Regressão com dados em painel com efeitos aleatórios. } \\
\mathrm{H}_{1} \text { : Regressão com dados e painel com efeitos fixos. }\end{array}$} \\
\hline $\begin{array}{c}\text { ECP } \\
\text { Teste de Hausman: } \\
\chi^{2}=13,98 \\
\text { Valor-P }=0,0516 \\
\text { Rejeita-se } \mathrm{H}_{0} \text { com significância } \\
\text { de } 5 \% \text {, mas não se rejeita com } \\
\text { significância de } 10 \% . \\
\text { Teste de Schaffer e Stillman: } \\
\text { Estatística Sargan-Hansen = } \\
10,19 \\
\text { Valor-p }=0,1778 \\
\text { Não se rejeita } \mathrm{H}_{0} \\
\text { Utilizar dados em painel com } \\
\text { efeitos aleatórios. }\end{array}$ & $\begin{array}{c}\text { ELP } \\
\text { Teste de Hausman: } \\
\chi^{2}=24,51 \\
\text { Valor-P }=0,0009 \\
\text { Rejeitar-se } \mathrm{H}_{0} \text { com significância } \\
\text { de } 1 \% \text {. } \\
\text { Utilizar dados em painel com } \\
\text { efeitos aleatórios. } \\
\text { Teste de Schaffer e Stillman: } \\
\text { Estatística Sargan-Hansen = } \\
\quad 38,78 \\
\text { Valor-p }=0,0000 \\
\text { Rejeita-se } \mathrm{H}_{0} \\
\text { Utilizar dados em painel com } \\
\text { efeitos fixos. }\end{array}$ & $\begin{array}{c}\text { Teste de Hausman: } \\
\chi^{2}=6,03 \\
\text { Valor-P }=0,5357 \\
\text { Não se rejeita } \mathrm{H}_{0 .} \\
\text { Utilizar dados em painel com } \\
\text { efeitos aleatórios. } \\
\text { Teste de Schaffer e Stillman: } \\
\text { Estatística Sargan-Hansen = } \\
7,56 \\
\text { Valor-p }=0,3753 \\
\text { Não se rejeita } \mathrm{H}_{0 .} \\
\text { Utilizar dados em painel com } \\
\text { efeitos aleatórios. }\end{array}$ \\
\hline
\end{tabular}

Fonte: Resultados da pesquisa.

Como se verifica na Tabela 2, os três modelos rejeitaram as respectivas hipóteses nulas do teste LM-Breusch-Pagan, confirmando a presença de efeitos longitudinais na amostra. Posteriormente, foram aplicados o Teste Robusto de Hausman e o Teste de Schaffer e Stillman, para selecionar o tipo de modelagem mais apropriada a cada caso: se painel com efeitos aleatórios ou com efeitos fixos.

Os resultados dos testes mencionados apontaram que a modelagem com dados em painel com efeitos aleatórios é mais adequada para o endividamento de curto prazo e o endividamento geral. Por outro lado, a regressão do endividamento de longo prazo apresenta melhor aderência à modelagem com dados em painel com efeitos fixos. Os resultados das regressões são tratados na Tabela 3. 
Ressalta-se que nos três modelos há significância conjunta das variáveis ao nível de 1\%, evidenciando que ao menos uma delas, em cada modelo, possui coeficiente estatisticamente diferente de zero, atestando a validade dos modelos em questão.

Tabela 3 - Resultados das regressões de endividamento de curto prazo, endividamento de longo prazo e endividamento geral

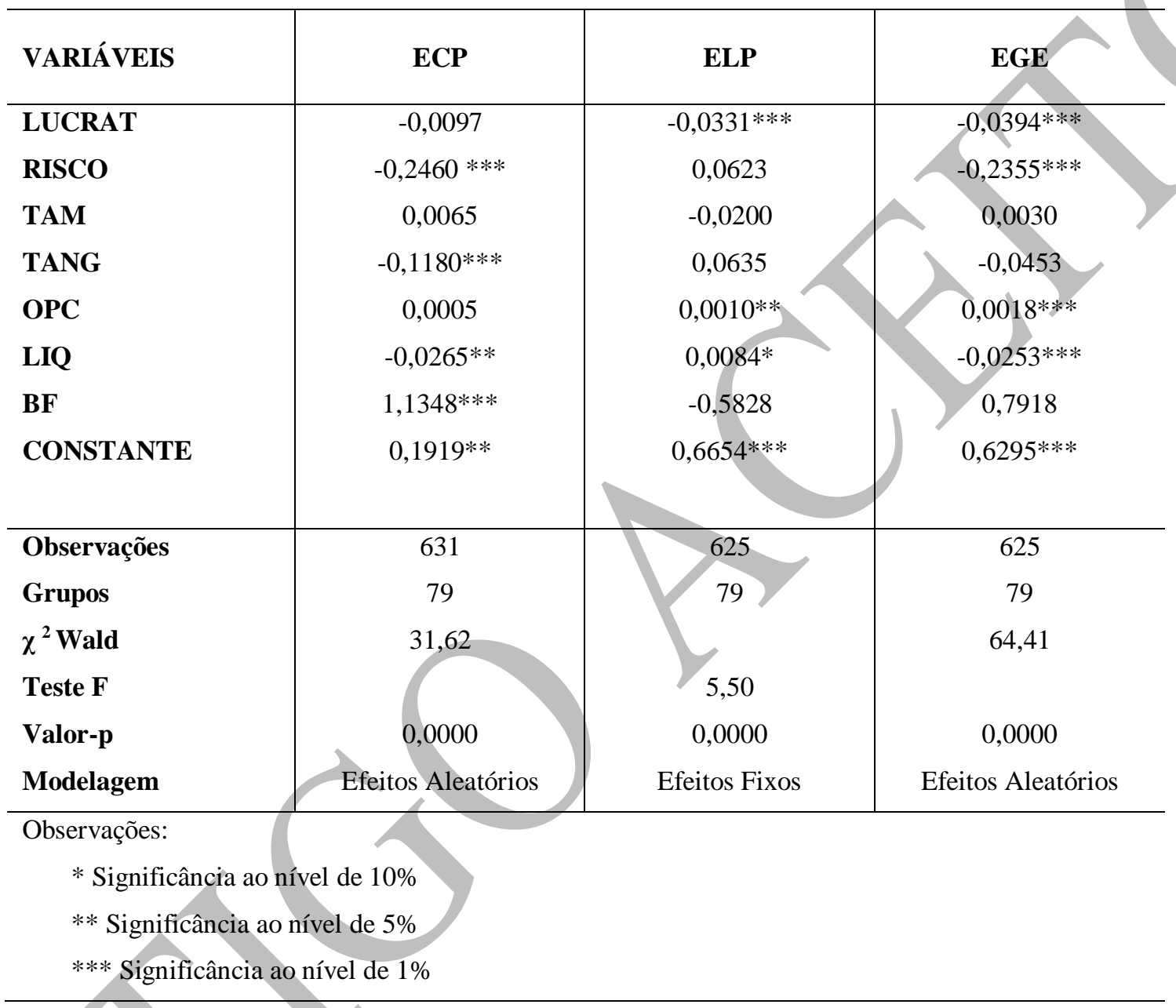

Fonte: Resultados da pesquisá.

De acordo com a Tabela 3, o coeficiente de lucratividade não apresentou significância estatística para o endividamento de curto prazo, mas evidenciou correlação negativa significante ao nível de $1 \%$ no endividamento de longo prazo e geral. A correlação negativa significante entre a lucratividade e o endividamento é um sinal característico da POT, apontando que os administradores preferem lucros retidos a endividamento para financiar novos projetos (Myers, 1984; Myers \& Majluf, 1984; Albanez \& Valle, 2009). Este resultado é coerente com os achados de Rajan and Zingales (1995), Booth et al., (2001), Perobelli e Famá (2002), Perobelli e Famá (2003), Shah and Hizaji (2004), Gaud et al., (2005), Huang and Song (2006), Correa et al., (2013), Vo (2017), Cevheroglu-Acar (2018), Jarallah et al., (2018), Haron (2018) e Kadek and Bagus (2019). 
A variável RISCO foi usada como proxy para o risco de falência e custos de agência, atrelados ao endividamento, indicando que empresas mais arriscadas sejam menos endividadas (Bradley, et al., 1984; Brito et al., 2007), sugerindo que esta seja negativamente correlacionada com o endividamento (Harris \& Raviv, 1991). As empresas com menores oscilações em seus resultados reduzem os riscos de dificuldades financeiras, podendo captar recursos a custos menores (Perobelli \& Famá, 2003). Os resultados mostraram correlação negativa e significante a $1 \%$ em relação ao endividamento de curto prazo e geral, comportando-se de acordo com o preconizado pela TOT e também pela POT. Os achados se mostraram congruentes com os resultados apurados por Gaud et al., (2005), Huang and Song (2006) e Cevheroglu-Acar (2018), embora contradigam os resultados obtidos por Brito et al., (2007) e Correa et al., (2013). O endividamento de longo prazo, por sua vez, não apresentou correlação significante em relação ao risco de falência.

Segundo a TOT, empresas maiores tendem a ser mais endividadas, em virtude de sua maior diversificação, o que as fazem menos expostas a riscos de falência, ensejando correlação positiva com o endividamento (Ferri \& Jones, 1979; Rajan \& Zingales, 1995; Titman \& Wessels, 1998). Por outro lado, Gaud et al., (2005) argumentam que tal relação deve ser negativa, pois as empresas menores possuem maior concentração acionária, aumentando os conflitos de agência, também de acordo com as premissas da TOT. Neste trabalho, as correlações entre o tamanho da firma e os níveis de endividamento não foram estatisticamente significantes.

Empresas detentoras de elevados volumes de ativos imobilizados podem reduzir seus custos de financiamento, pois estes ativos funcionam como garantias em relação às dívidas, permitindo maior endividamento, o que sugere correlação positiva entre endividamento e tangibilidade dos ativos (Perobelli \& Famá, 2003; Rajan \& Zingales, 1995). Entretanto, Brito et al., (2007) sugerem que a correlação seja negativa com o endividamento de curto prazo. Os resultados obtidos encontraram correlação negativa e significante ao nível de $1 \%$ entre o endividamento de curto prazo e a tangibilidade, não havendo correlação significante com o endividamento de longo prazo e o geral. Embora contrariando as hipóteses da POT e TOT, os resultados apurados neste trabalho são semelhantes aos encontrados por Brito et al., (2007) e Bastos et al., (2009), em relação ao endividamento de curto prazo.

A variável OPC é uma proxy para as oportunidades de crescimento da firma, utilizada em trabalhos empíricos de Titman and Wessels (1998), Gaud et al., (2005), Brito et al., (2007), Bastos et al., (2009) e Albanez e Valle (2009), dentre outros. As empresas com maiores 
oportunidades de crescimento têm mais opções para investimento, incorrendo em custos de agência e custos de falência potencialmente menores (Brito et al., 2007), gerando ativos intangíveis que não servem como garantia de dívidas (Correa, et al., 2013). Segundo Hovakimian, Opler and Titman (2001), as empresas com oportunidades favoráveis de crescimento tendem a valorizar as suas ações, desestimulando o endividamento. Espera-se correlação negativa com o endividamento na TOT e positiva ou negativa, na POT (Bastos et al., 2009).

Os resultados apontaram correlação positiva em relação ao endividamento de longo prazo e geral, com significância de 5\% e 1\%, respectivamente, inexistindo correlação significante com o endividamento de curto prazo. A recomposição tarifária automática, prevista pela Agência Nacional de Energia Elétrica - ANEEL, pode justificar a tendência apontada, ao garantir a correção automática do faturamento das empresas ao longo do tempo. Embora divergentes, estes resultados têm alinhamento com a POT, de acordo com os pressupostos teóricos de Jensen (1986) e os achados de Brito et al., (2007). Segundo Correa et al., (2013), empresas com elevado potencial de crescimento podem necessitar de recursos adicionais, captados via incremento no endividamento, justificando os resultados empiricamente alcançados neste trabalho.

Liquidez corrente é a capacidade de pagamento pontual das dívidas de curto prazo da empresa (Assaf, 2012), tendo LIQ como proxy. É previsto que o endividamento e liquidez sejam negativamente correlacionados, segundo a POT e assumam correlação positiva ou negativa na TOT (Bastos et al., 2009; Vo, 2017). Os resultados apontaram correlação negativa e significante ao nível de $5 \%$ e $1 \%$ em relação às dívidas de curto prazo e geral e positiva significante ao nível de $10 \%$ com o endividamento de longo prazo.

Os resultados corroboram as tendências tradicionais da POT no curto prazo e em relação ao endividamento geral, mas são incoerentes com a evidência dominante no longo prazo. Empresas com boa liquidez usam mais fundos internos para financiar seus projetos, segundo a POT (Haron, 2018), enquanto Cevheroglu-Acar (2018) afirma que empresas com maior liquidez podem se endividar mais, em virtude da sua maior capacidade para cumprir obrigações de curto prazo, de acordo com a TOT. Uma provável explicação para a tendência encontrada se apoia no elevado custo de captação de recursos no mercado brasileiro, que pode desestimular o financiamento via endividamento. 
Os resultados apurados em relação à liquidez foram consistentes com os achados para o Brasil de Bastos et al., (2009), de Cevheroglu-Acar (2018) em relação ao curto prazo e de Rodrigues et al., (2017) e Haron (2018), em relação ao endividamento de longo prazo.

A variável BF funciona como proxy do benefício fiscal gerado pelo endividamento e, por isso, empresas com maiores taxas de depreciação e elevados níveis de investimento fixo tendem a ser menos endividadas (Titman \& Wessels, 1988; Frank \& Goyal, 2009), segundo as premissas da TOT (Haron, 2018).

Os resultados apontaram relação positiva significante ao nível de $1 \%$ para o endividamento de curto prazo, inexistindo significância estatística para o endividamento de longo prazo e o geral. Estes resultados, embora contrários à evidência da TOT, podem ser explicados pela característica de imobilização de capital, própria do setor. Os achados empíricos deste trabalho são coerentes com os obtidos por Bradley et al., (1984), Rajan and Zingales (1995), Huang and Song (2006) e Haron (2018), sinalizando que as empresas detentoras de volumes elevadas de ativos fixos tendem a se endividarem mais.

\begin{tabular}{|c|c|c|c|c|}
\hline Variável & $\begin{array}{c}\text { Correlação } \\
\text { Esperada }\end{array}$ & ECP & ELP & EGE \\
\hline LUCRAT & $\begin{array}{l}\text { TOT }(+) \\
\text { POT (-) }\end{array}$ & Não significante & $\begin{array}{c}\text { Negativa } \\
\text { Significância: } 1 \%\end{array}$ & $\begin{array}{c}\text { Negativa } \\
\text { Significância: } 1 \%\end{array}$ \\
\hline RISCO & $\begin{array}{l}\text { TOT (-) } \\
\text { POT (-) }\end{array}$ & $\begin{array}{c}\text { Negativa } \\
\text { ignificância: } 1 \%\end{array}$ & Não Significante & $\begin{array}{c}\text { Negativa } \\
\text { Significância: } 1 \%\end{array}$ \\
\hline & $\begin{array}{c}\text { TOT (+) } \\
\text { POT (+ ou -) }\end{array}$ & Não significante & Não significante & Não significante \\
\hline & $\begin{array}{l}\text { TOT (+) } \\
\text { POT (+) }\end{array}$ & $\begin{array}{c}\text { Negativa } \\
\text { Significância: } 1 \%\end{array}$ & Não significante & Não significante \\
\hline OPC & $\begin{array}{c}\text { TOT (-) } \\
\text { POT (+ ou -) }\end{array}$ & Não significante & $\begin{array}{c}\text { Positiva } \\
\text { Significância: } 5 \%\end{array}$ & $\begin{array}{c}\text { Positiva } \\
\text { Significância: } 1 \%\end{array}$ \\
\hline LIQ & $\begin{array}{l}\text { TOT (não } \\
\text { consta) } \\
\text { POT (-) }\end{array}$ & $\begin{array}{c}\text { Negativa } \\
\text { Significância: } 5 \%\end{array}$ & $\begin{array}{c}\text { Positiva } \\
\text { Significância: } 10 \%\end{array}$ & $\begin{array}{c}\text { Negativa } \\
\text { Significância: } 1 \%\end{array}$ \\
\hline BENFND & $\begin{array}{l}\text { TOT (-) } \\
\text { POT (Não } \\
\text { consta) }\end{array}$ & $\begin{array}{c}\text { Positiva } \\
\text { Significância: } 1 \%\end{array}$ & Não significante & Não significante \\
\hline
\end{tabular}


Figura 2: Correlações esperadas e resumo dos resultados obtidos. (Fonte: Resultados da pesquisa).

\section{CONSIDERAÇÕES FINAIS}

O objetivo deste trabalho foi estudar os determinantes da estrutura de capital das empresas de capital aberto do setor elétrico brasileiro, relativos ao endividamento de curto prazo, longo prazo e geral, entre 2009 e 2019, a partir das premissas da TOT e da POT. Os determinantes de estrutura de capital avaliados foram o impacto da lucratividade, risco de falência, tamanho, tangibilidade, oportunidades de crescimento, liquidez e benefícios fiscais não decorrentes de dívidas. As variáveis dependentes usadas foram o endividamento de curto prazo, endividamento de longo prazo e o endividamento geral.

Os resultados apontaram que os determinantes de lucratividade, oportunidades de crescimento e liquidez tiveram correlação com os endividamentos estudados em melhor alinhamento com as proposições da POT, enquanto o risco se comportou de acordo com as premissas da TOT e da POT. Destaca-se que o benefício fiscal não decorrente de dívidas apresentou correlação oposta àquela preconizada pela TOT, o que pode ser apontado como um achado relevante da pesquisa, tendo em vista o seu comportamento contrário ao esperado. Os resultados mostraram que empresas que possuíam mais benefícios fiscais seriam mais endividadas, ao menos no curto prazo.

Portanto, à luz dos achados, conclui-se que as empresas de capital aberto do setor elétrico brasileiro tiveram seus determinantes de estrutura de capital melhor explicados pela POT, revelando que as tendências de assimetria informacional e hierarquia de fontes de financiamento foram relevantes.

A partir dos resultados obtidos, há indicação de que as empresas de capital aberto do setor elétrico brasileiro tendem a preferir financiar os seus novos projetos usando preferencialmente recursos internos, como os lucros retidos e, se não forem suficientes, recorrem ao endividamento. A emissão de novas ações seria utilizada se o financiamento com dívidas não fosse suficiente.

Entretanto, estes resultados devem ser tratados com a devida parcimônia, em razão da estrutura amostral utilizada, a qual impôs limitações à pesquisa. O trabalho considerou apenas dados financeiros de empresas de capital aberto, em virtude de sua disponibilidade. 
Entretanto, ao se considerar o universo amostral composto pelas empresas do setor elétrico brasileiro, a supressão de firmas de capital fechado gera viés de seleção, o que compromete a generalização dos resultados aqui alcançados.

Por outro lado, em que pesem as limitações apontadas, este trabalho procura estabelecer contribuições teóricas, ao lançar luzes sobre a estratégia e preferências de financiamento das empresas de capital aberto, atuantes no setor elétrico brasileiro. Considerando-se a relevância do setor para a economia nacional, a compreensão desta dinâmica de comportamento pode ser importante para tornar o setor elétrico um ambiente ainda mais competitivo, com empresas financeiramente saudáveis, capazes de prestar serviços de alta qualidade, atendendo aos anseios do mercado e dos consumidores. Este trabalho também trouxe contribuição prática, ao prover a aplicação de métodos quantitativos específicos para a identificação dos determinantes da estrutura de capital das empresas de capital aberto do setor elétrico brasileiro. Estas ferramentas trazem indicativos para a avaliação do endividamento das firmas e, consequentemente, elementos para a análise da performance financeira das empresas do setor.

Como sugestão para futuras pesquisas, indica-se a replicação desta metodologia a outros setores econômicos, considerando períodos temporais mais amplos, para verificar a manutenção das tendências encontradas para outros ramos de atividade. Com a finalidade de mitigar os efeitos do viés de seleção, a inclusão de empresas de capital fechado seria importante, levando-se em conta, entretanto, as dificuldades para a obtenção de dados deste grupo de empresas. Adicionalmente, sugerem-se pesquisas envolvendo empresas do setor elétrico operantes na América Latina, com a finalidade de se avaliarem os comportamentos dos determinantes de estrutura de capital em âmbito continental.

Também merece destaque a ausência de correlação significante do endividamento com o tamanho das empresas. Por se tratarem de empresas que operam em um ambiente específico, regulamentado por agência própria, estudos mais aprofundados seriam desejáveis em trabalhos futuros, para melhor investigar esta particularidade.

\section{REFERÊNCIAS}

Albanez, T., \& Valle, M. R. (2009). Impactos da assimetria de informação na estrutura de capital de empresas brasileiras abertas. Revista de Contabilidade \& Finanças, 20(51), 6-27.

Assaf, A., Neto. (2012). Finanças corporativas e valor (6 ed.). São Paulo: Atlas. 
Aydin, M. (2019). The effect of biomass energy consumption on economic growth in BRICS countries: a country-specific panel data. Renewable Energy, 138, 620-627.

Bagais, O., Aljaaidi, K., \& Alothman, A. (2021). An empirical investigations of the associations of short and long term debt policies and economic values of energy sector. International Journal of Energy Economics and Policy, 11(1), 249-254.

Baltagi, B. H. (1998). Econometrics. New York: Springer.

Bastos, D. D., Nakamura, W. T., \& Basso, L. C. (2009). Determinantes da estrutura de capital das companhias abertas na América Latina: um estudo empírico considerando fatores macroeconômicos e institucionais. Revista de Administração Mackenzie, 10(6), 47-77.

Bastos, D. D., \& Nakamura, W. T. (2009). Determinantes da estrutura de capital das companhias abertas no Brasil, México e Chile no período 2001-2006. Revista de Contabilidade e Finanças, 20(50), 75-94.

Belke, A., Dreger, C., \& Haan, F. (2010). Energy consumption and economic growth: new insights into the cointegration relationship. Ruhr Economic Papers. Retrieved May, 04, 2021, from http://hdl.handle.net/10419/37017.

Bhama, V., Jain, P. K., \& Yadav, S. S. (2019). Pecking order test at varying debt levels: a comparative study of Indian and Chinese firms. Journal of Emerging Markets Finance, 18(2), 237-261.

Booth, L., Aivazian. V., Demirguc-Kunt, A., \& Maksimovic, V. (2001). Capital structures in developing countries. The Journal of Finance, 41(1), 87-130.

Bradley, M., Jarrel, G. A., \& Kim, E. H. (1984). On the existence of an optimal capital structure: theory and evidence. American Finance Association, 39(3), 857-878.

Brealey, R. A., Myers, S. C., \& Allen, F. (2013). Princípios de finanças corporativas. Porto Alegre: AMGH Editora.

Brito, G. A., Corrar, L.J., \& Batistella, F. D. (2007). Fatores determinantes da estrutura de capital das maiores empresas que atuam no Brasil. Revista de Contabilidade e Finanças, 43, 9-19.

Cameron, A. A., \& Trivedi, P. K. (2010). Microeconometrics using Stata. New York: The Stata Press, 2010.

Cervo, A. L., \& Bervian, P. A. (2002). Metodologia científica (5 ed.). São Paulo: Pearson Prentice Hall.

Cevheroglu-Acar, M. G. (2018). Determinants of capital structure: evidence from Turkey. Journal of Management and Sustainability, 8(1), 31-45.

Correa, C. A., Basso, L. F., \& Nakamura, W. T. (2013). A estrutura de capital das maiores empresas brasileiras: análise empírica das teorias de Pecking Order e Trade-Off, usando panel data. Revista de Administração Mackenzie, 14(4), 106-133.

DeAngelo, H., \& Masulis, R. W. (1980). Optimal capital structure under corporate and personal taxation. Journal of Financial Economics, 8, 3-39.

Doege, R., \& Matos, R. A. (2011). Estrutura de capital: investigação de um padrão de comportamento nas distribuidoras de energia elétrica. Anais do XVIII Congresso Brasileiro de Custos, Rio de Janeiro, RJ, Brasil. 
Espinosa, M. C., Maquieira, C. V., Vieito, J. P., \& González, M. A. (2012). Capital structures in developing countries: the Latin American case. Investigación Económica, 61(282), 35-54.

Ferri, M., \& Jones, W. (1979). Determinants of financial structure: a new methodological approach. Journal of Finance, 34, 631-644.

Ferreira, R. M., Bertucci, L. A., \& Pereira, A. D., Filho. (2010). Relação entre estrutura de capitais e estrutura de ativos nos setores brasileiros de energia elétrica e telecomunicações. Revista Brasileira de Gestão de Negócios, 12(34), 7-24.

Fotourehchi, Z. (2017). Renewable energy consumption and economic growth: a case study for developing countries. International Journal of Energy Economics and Policy, 7(2), 62-64.

Frank, M. Z., \& Goyal, V. K. (2009). Capital structure decisions: which yariables are realibly important? Financial Management, 38(1), 1-37.

Ganim, A. (2008). O setor elétrico brasileiro: aspectos regulamentares, tributários e contábeis. Brasília: Synergia.

Gaud, P., Jani, E., Hoesli, M., \& Bender, A. (2005). The capital structure of Swiss companies: na empirical analysis using panel data. European Financial Management, 11(1), 51-69.

Goldemberg, J., \& Lucon, O. (2008). Energia, meio ambiente e desenvolvimento. São Paulo: EDUSP.

Greene, W. H. (2008). Econometric analysis. New Jersey: Pearson Prentice Hall.

Hair, J. F., Black, W. C., Babin, B. J., Anderson, R. E., \& Tatham R. L. (2009). Análise multivariada de dados. Porto Alegre: Bookman.

Haron, R. (2018). Firm level, ownership concentration and industry level determinants of capital structure in na emerging market: Indonesia evidence. Asian Academy of Management Journal of Accounting and Finance, 14(1), 127-151.

Harris, M., \& Raviv, A. (1991). The theory of capital structure. The Journal of Finance, 46(1), 297-355.

Hovakimian, A., Opler, T., \& Titman, S. (2001). The debit-equity choice. Journal of Financial and Quantitative Analysis, 36(1), 1-24.

Huang, S. G., \& Song, F. M. (2006). The determinants of capital structure: evidence from China. China Economic Review, 17, 14-36.

Jarallah, S., Saleh, A. S., \& Salim, R. (2019). Examining pecking order versos trade-off theories of capital structure: new evidences from Japanese firms. International Journal of Finance and Economics, 24, 204-211.

Jensen, M. (1986). Agency costs of free cash flow, corporate finance and takeovers. The American Economic Review, 6(2), 326-329.

Jensen, M., \& Meckling, W. (1976). Theory of the firm: managerial behavior, agency costs and ownership structure. Journal of Financial Economics, 3(4), 305-360.

Kadek, E. M., Bagus, A. P. (2019). Capital structure variables of pecking order theory perspective in Indonesia stock exchange. Russian Journal of Agricultural and SocioEconomic Sciences, 11(95), 111-121.

Kennedy, P. (2009). Manual de econometria. Rio de Janeiro: Campus. 
Kuroda, A., Moralles, H. F., \& Albuquerque, A. A. (2019). The effects of financial leverage and debt maturity on the investments of Brazilian electric sector companies. Revista Brasileira de Gestão de Negócios, 21(3), 563-581.

Leland, H. E., \& Pyle, D. H. (1977). Informational asymmetries, financial structure and financial intermediation. The Journal of Finance, 32(2), 371-387.

Lima, M. R., \& Brito, R. D. (2010). O que determina a estrutura de capital no Brasil? Anais do X Encontro Brasileiro de Finanças, São Paulo, SP, Brasil.

Martinez, L. B., Scherger, V. \& Guercio, M. B. (2019). SMEs capital structure: trade-off or pecking order theory: a systematic review. Journal of Small Business and Enterprise Development, 26(1), 105-132.

Md-Yusuf, M., Yunus, F. M., \& Supaat, N. Z. (2013). Determinants of capital structure in Malaysia electrical and electronic sector. International Journal of Social, Behavioral, Educacional, Economic, Business and Industrial Engeneering, 7(6), 1514-1519.

Miller, M. H. (1977). Debt and taxes. The Journal of Finance, 32(2), 261-275.

Modigliani, F., \& Miller, M. (1958). The costs of capital, corporation finance, and the theory of investment. The American Economic Review, 48(3), 261-297.

Modigliani, F., \& Miller, M. (1963). Corporate income taxes and the cost of capital: a correction. The American Economic Review, 53(3), 433-443.

Montoya, M. A., Pasqual, C. A., Lopes, R. L., \& Guilhoto, J. J. (2013). As relações intersetoriais do setor energético no crescimento da economia brasileira: uma abordagem insumo-produto. Recuperado em 06 maio, 2021, de http://www.usp.br/nereus/wpcontent/uploads/TD_Nereus_12_2013b.pdf.

Mudany, J. O., Letting, N. K., \& Gituro, W. (2020). Intervening effects of capital structure on strategy implementation and performance of energy sector institutions in Kenya. Journal of Finance and Accounting, 4(3), 73-95.

Myers, S. C. (1984). The capital structure puzzle. The Journal of Finance, 39(3), 575-592.

Myers, S. C. (2001). Capital structure. The Journal of Economic Perspectives, 15(2), 81-102.

Myers, S. C., \& Majluf, N. S. (1984). Corporate financing and investment decisions when firms have information that investors do not have. National Bureau of Economic Research, [Working Paper Series n. 1.396]. Retrieved December 11, 2020, from https://www.nber.org/system/files/working_papers/w1396/w1396.pdf.

Niu, S., Jia, Y., Wang, W., He, R., Hu, L., \& Liu, Y. (2013). Electric consumption and human development level: a comparative analysis based on panel data for 50 countries. Electric Power and Energy Systems, 53, 338-347.

Ouedraogo, N. S. (2013). Energy consumption and human development: evidence from a panel cointegration and error correction model. Energy, 63(15), 28-41.

Ozkan, A. (2001). Determinants of capital structure and adjustment to long run target: evidence from UK company panel data. Journal of Business, Finance and Accounting, 1(2), 175-198.

Perobelli, F. F., \& Famá, R. (2002). Determinantes da estrutura de capital: aplicação a empresas de capital aberto brasileiras. Revista de Administração, 37(3), 33-46. 
Perobelli, F. F., \& Famá, R. (2003). Fatores determinantes da estrutura de capital para empresas latino-americanas. Revista de Administração Contemporânea. 7(1), 9-35.

Pindyck, R. S., \& Rubinfeld, D. L. (2004). Econometria: modelos e previsões. Rio de Janeiro: Elsevier.

Rajan, R. G., \& Zingales, R. (1995). What do we know about capital structure? Some evidence from international data. Journal of Finance, 50, 1421-1460.

Rodrigues, S. V., Moura, H. J., Santos, D. F., \& Sobreiro, V. A. (2017). Capital structure management differences in Latin American and US firms after 2008 crisis. Journal of Economics, Finance and Administrative Science, 22(42), 51-74.

Ross, S. (1977). The determination of financial structure: the incentive signalling approach. Bell Journal of Economics, 8, 23-40.

Sant'Ana, C. F., \& Silva, T. P. (2015). Fatores determinantes da estrutura de capital de empresas brasileiras de tecnologia. Journal of Information Systems and Technology Management, 12(3), 687-708.

Serva, M. (2003). Análise de empresas privatizadas: o desafio da multidimensionalidade. Civitas, 3(2), 349-373.

Shah, A., \& Hizaji, T. (2004). The determinants of capital structure of stock exchange-listed non-financial firms in Pakistan. The Pakistan Development Review, 43(4), 605-618.

Shyam-Sunder, L., \& Myers, S. C. (1999). Testing static tradeoff against Pecking order models of capital structures. Journal of Financial Economics, 51, 219-244.

Spiegel, Y., \& Spulber, D. F. (1994). The capital structure of a regulated firm. RAND Journal of Economics, 25(3), 424-440.

Tiryaki, G. F. (2017). Análise de dados em painel. In: Malbouisson, C., \& Tiryaki, G. F. Econometria na prática. Rio de Janeiro: Alta Books.

Titman, S. (1984). The effect of capital structure on a firm's liquidation decisions. Journal of Financial Economics. 13(1), 137-151.

Titman, S., \& Wessels, R. (1988). The determinants of capital structure choice. The Journal of Finance. 18(1), 1-19.

Triola, M. F. (2012). Introdução à estatística. (10. ed.). Rio de Janeiro: LTC.

Tsaurai, K., \& Ngcobo, L. (2020). Renewable energy consumption, education and economic growth in Brazil, Russia, India, China, South Africa. International Journal of Energy Economics and Policy, 10(2), 26-34.

Vo, X. (2017). V. Determinants of capital structure in emerging markets: evidence from Vietnam. Research in International Business and Finance, 40, 105-113.

Wooldridge, J. M. (2016). Introdução à econometria: uma abordagem moderna. São Paulo: Cengage Learning. 